\section{International Scientific Journal Theoretical \& Applied Science}

\author{
p-ISSN: 2308-4944 (print) e-ISSN: 2409-0085 (online) \\ Year: $2016 \quad$ Issue: 4 Volume: 36 \\ Published: $30.04 .2016 \quad \underline{\text { http://T-Science.org }}$
}

SECTION 20. Medicine.

\begin{abstract}
Anna Evgenevna Shulgan
$\mathrm{PhD}$, Medical doctor, postgraduate student of clinical psychology, Institute of psychotherapy and clinical psychology (Moscow), Russia dr.shulgan@gmail.com

Vladimir Igorevich Esaulov Institute of psychotherapy and clinical psychology (Moscow), Psychotherapy Department, Senior Lecturer, Assistant, Department of Psychotherapy, Pirogov Russian National Research Medical

University, Russia v-esaulov@yandex.ru
\end{abstract}

Alexey Vasilevich Borsukov

Doctor of Medical Sciences, Full Professor, Head of research laboratory "Diagnostics and minimally invasive technologies " Smolensk State Medical Academy, head of the city department of diagnostic and minimally invasive technologies "Clinical Hospital №1» of Smolensk, Russia

bor55@yandex.ru

\title{
PSYCHOLOGICAL CHARACTERISTICS OF PATIENTS WITH DIFFUSE LIVER DISEASES
}

\begin{abstract}
Psycho-emotional disorders, associated to diffuse liver diseases, contributing it significantly and create difficulties in its treatment. In this research was examined the psychological characteristics of patients with diffuse liver diseases using of Hospital Anxiety and Depression Scale (HADS). Total examined 141 patients with diffuse liver diseases - 56 women (39.72\%) and 85 men (60.28\%). The research revealed that the absence of anxiety is a typical symptom for male patients with non-alcoholic steatohepatitis. Subclinical anxiety is more common for female patients with alcoholic steatohepatitis, cirrhosis of class A and class B of Child-Pugh, as well as in men with alcoholic steatohepatitis and liver cirrhosis class A Child-Pugh. Clinically significant anxiety is typical of patients with chronic viral hepatitis. Subclinical depression is a typical sign for women suffering from cirrhosis of the class C Child-Pugh and for male patients with cirrhosis Class B Child-Pugh. Clinical depression is characteristic of patients with alcoholic steatohepatitis.

Key words: diffuse liver diseases, anxiety, depression, Hospital Anxiety and Depression Scale (HADS).

Language: Russian

Citation: Shulgan AE, Esaulov VI, Borsukov AV (2016) PSYCHOLOGICAL CHARACTERISTICS OF PATIENTS WITH DIFFUSE LIVER DISEASES. ISJ Theoretical \& Applied Science, 04 (36): 184-189.

Soi: http://s-o-i.org/1.1/TAS-04-36-31 Doi: crossef http://dx.doi.org/10.15863/TAS.2016.04.36.31 \section{ПЕЧЕНИ}

ПСИХОЛОГИЧЕСКИЕ ОСОБЕННОСТИ У ПАЦИЕНТОВ С ДИФФУЗНЫМИ ЗАБОЛЕВАНИЯМИ

Аннотация: Психоэмоциональные расстройства, сопутствующчие диффузным заболеваниям печени, значительно усугубляют их течение и создают трудности в лечении. В настоящем исследовании изучались психологические особенности пациентов с диффузными заболеваниями печени с помощью госпитальной шкаль тревоги и депрессии (HADS). Всего обследован 141 человек с диффузными заболеваниями печени 56 женщин (39,72\%) и 85 мужчин (60,28\%). В результате исследования выявлено, отсутствие тревоги является типичным признаком для пациентов мужского пола с неалкогольным стеатогепатитом. Субклинически выраженная тревога чаще встречается у женщин с алкогольным стеатогепатитом, циррозом печени класса A и класса B по Child-Pugh, а так же у мужчин с алкогольным стеатогепатитом u циррозом печени класса A по Child-Pugh. Клинически выраженная тревога типична для пациенток $c$ хроническим вирусным гепатитом. Субклиническая депрессия является типичным признаком для женшин, страдающих ичррозом печени класса C по Child-Pugh и для пациентов мужского пола с ииррозом печени
\end{abstract}


класса B по Child-Pugh. Клиническая депрессия характерна для пациченток с алкогольным стеатогепатитом.

Ключевые слова: диффузные заболевания печени, тревога, депрессия, госпитальная шкала тревоги и депрессии (HADS).

Во многих исследованиях, посвященных изучению коморбидности при различных соматических заболеваниях, отмечено, что сопутствующие основной симптоматике психоэмоциональные расстройства могут существенно поменять течение заболевания, а нередко и усугубить её тяжесть. Это в свою очередь может затруднять лечение и нередко создаёт препятствия для проведения диагностических мероприятий [1-6].

Имеется ряд клинических наблюдений, описывающих возникновение различных психических нарушений при заболеваниях печени [7-12]. Печень участвует в разнообразных метаболических процессах, что определяет её уязвимость и является предпосылкой для сложного патогенеза поражений нервной системы [13, 14]. Особенно это становится заметным в тех случаях, когда при различных стрессовых воздействиях функционирование как нервной системы, так и печени может быть серьезно нарушено [15-18].

Целью работы явилось исследование психологических особенностей у пациентов с диффузными заболеваниями печени.

Материал и методы исследования: Всего был обследован 141 человек с диффузными заболеваниями печени - 56 женщин $(39,72 \%)$ и 85 мужчин (60,28\%). Из них 30 пациентов имели диагноз хронический вирусный гепатит (ХВГ), 67 - стеатогепатит (48 - алкогольный (АСГ), 19 неалкогольный (НАСГ)), и 44 - цирроз печени различного генеза (ЦП). Оценка тяжести цирроза печени осуществлялась с помощью международной классификации Чайлд-Пью (Child-Pugh). Количество пациентов с компенсированным циррозом печени (класс А) составило 22 человека, с субкомпенсированным циррозом печени (класс В) - 14 человек, с декомпенсированным циррозом печени (класс С) 8 человек.

Каждый больной подвергался полному клиническому обследованию, проводился анализ лабораторно-инструментальных

данных. Перечень обязательных исследований и лечения был в полном соответствии со стандартами (протоколами) приказа Министерства здравоохранения РФ (приказы Министерства здравоохранения и социального развития РФ №571 от 21.07.2006 г. и №811 от 27.12.2007 г.; приказы министерства здравоохранения РФ № 669н и №685н от 7.11.2012, №772н и №786н от 9.11.2012, №906н от12.11.2012). Для определения психологического статуса пациентов использовалась госпитальная шкала тревоги и депрессии (HADS), использованной для первичного выявления (скрининга) тревоги и депрессии у обследованных.

Для статистического анализа данных использовалась среда «R». Статистический анализ данных проводился методами описательной статистики и сравнения выборок. Для определения типичности проявления признаков внутри каждой группы мы использовали точный биномиальный критерий Фишера. Межгрупповые различия в распределении частот оценивали с помощью углового преобразования Фишера.

Результаты исследования: При анализе частот показателя A (тревога) шкалы HADS выявлено, что типичным признаком для женщин с циррозом печени класса А и В по Child-Pugh и алкогольным стеатогепатитом является субклиническая тревога $(\mathrm{p}<0,05)$. Для пациенток с ХВГ типичным признаком является клинически выраженная тревога $(\mathrm{p}<0,05)$. У женщин $\mathrm{c}$ циррозом печени класса $\mathrm{C}$ по Child-Pugh и неалкогольным стеатогепатитом с равной вероятностью можно встретить все три состояния $(\mathrm{p}>0,05)$. Показатели тревоги у женщин представлены на рисунке 1 (степень выраженности тревоги указана в условных единицах). 


\begin{tabular}{|c|c|c|c|c|c|c|}
\hline Impact Factor: & $\begin{array}{l}\text { ISRA (India) } \\
\text { ISI (Dubai, UAE } \\
\text { GIF (Australia) } \\
\text { JIF }\end{array}$ & $\begin{array}{l}=1.344 \\
=0.829 \\
=0.564 \\
=1.500\end{array}$ & $\begin{array}{l}\text { SIS (USA) } \\
\text { PИНЦ (Russia) } \\
\text { ESJI (KZ) } \\
\text { SJIF (Morocco) }\end{array}$ & $\begin{array}{l}=0.912 \\
=0.234 \\
=1.042 \\
=\mathbf{2 . 0 3 1}\end{array}$ & $\begin{array}{l}\text { ICV (Poland) } \\
\text { PIF (India) } \\
\text { IBI (India) }\end{array}$ & $\begin{array}{l}=6.630 \\
=1.940 \\
=4.260\end{array}$ \\
\hline
\end{tabular}

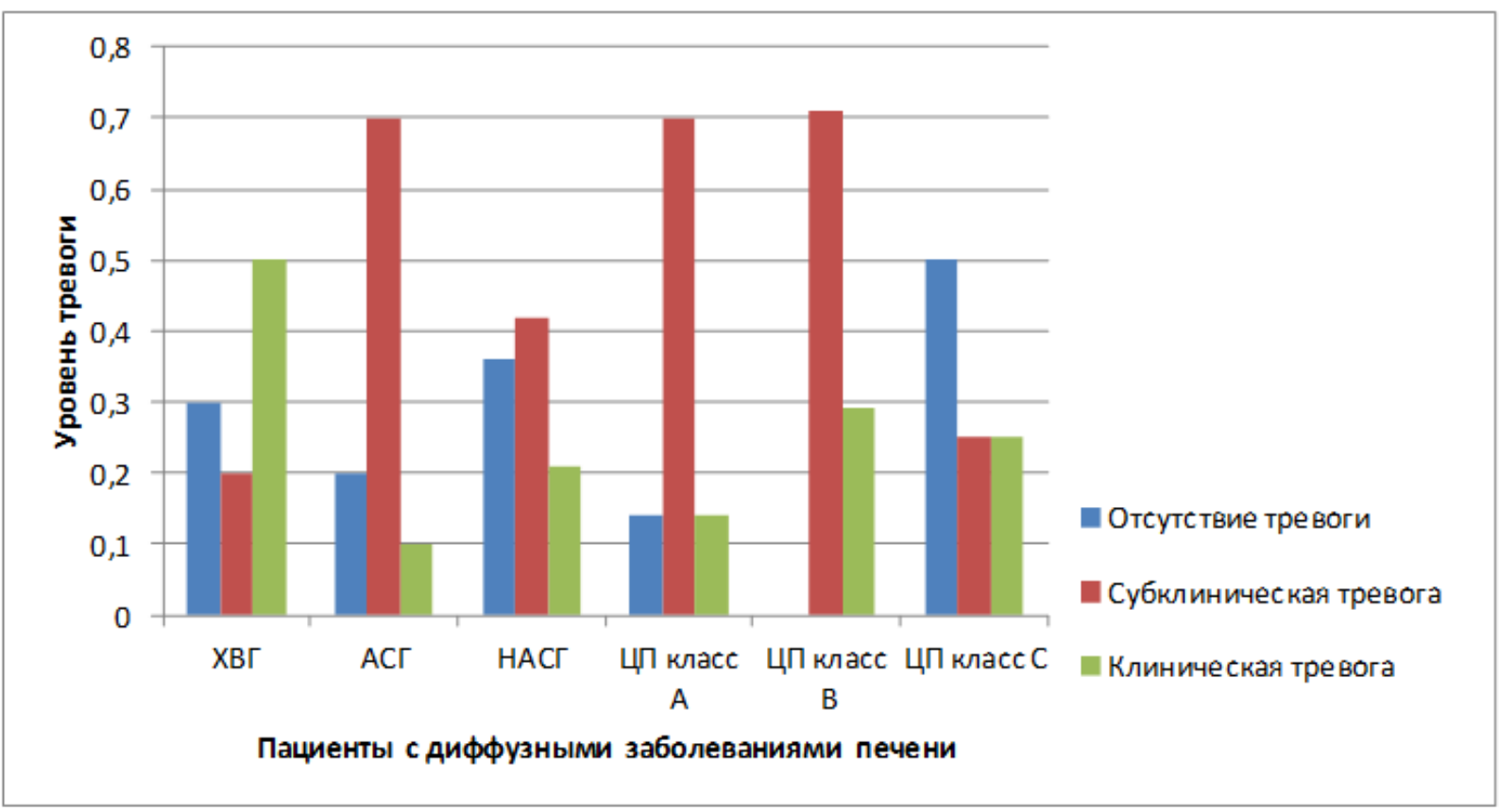

Рисунок 1 - Показатели тревоги у женщин.

Типичным признаком для мужчин с циррозом печени класса А по Child-Pugh и алкогольным стеатогепатитом является субклиническая тревога $(\mathrm{p}<0,05)$. У мужчин с ЦП класса $\mathrm{C}$ по Child-Pugh с равной вероятностью можно встретить состояния клинически и субклинически выраженной тревоги (p>0,05), а у больных с ЦП класса В по Child-Pugh и ХВГ все три состояния $(\mathrm{p}>0,05)$. Для пациентов c неалкогольным стеатогепатитом типичным признаком является отсутствие тревоги $(\mathrm{p}<0,05)$. Показатели тревоги у мужчин представлены на рисунке 2 (степень выраженности тревоги указана в условных единицах).

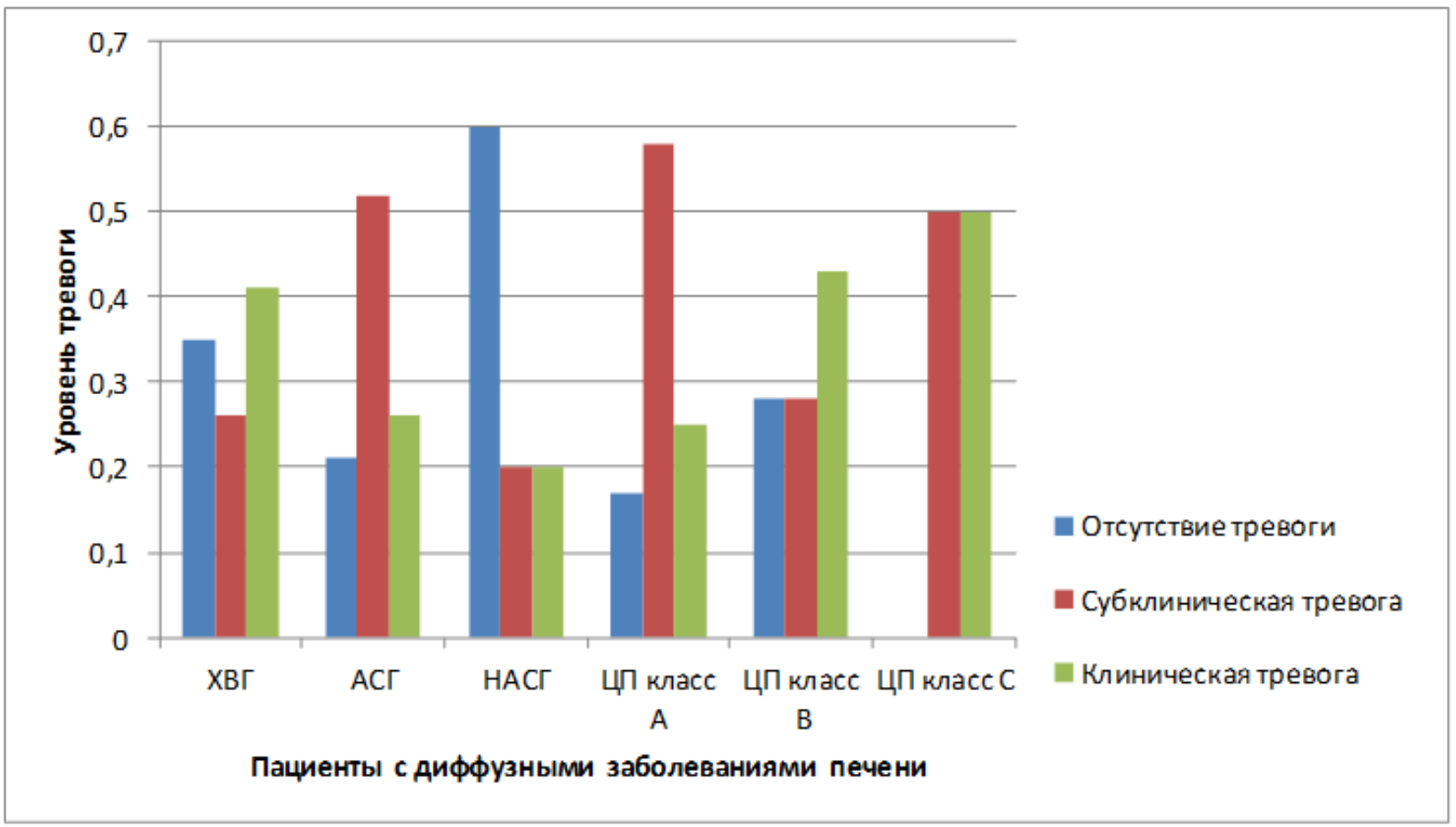

Рисунок 2 - Показатели тревоги у мужчин.

ISPC Global Science, 


\begin{tabular}{|c|c|c|c|c|c|c|}
\hline Impact Factor: & $\begin{array}{l}\text { ISRA (India) } \\
\text { ISI (Dubai, UAB } \\
\text { GIF (Australia) } \\
\text { JIF }\end{array}$ & $\begin{array}{r}=1.344 \\
=0.829 \\
=0.564 \\
=1.500\end{array}$ & $\begin{array}{l}\text { SIS (USA) } \\
\text { PИHЦ (Russia) } \\
\text { ESJI (KZ) } \\
\text { SJIF (Morocco) }\end{array}$ & $\begin{array}{l}=0.912 \\
=0.234 \\
=1.042 \\
=2.031\end{array}$ & $\begin{array}{l}\text { ICV (Poland) } \\
\text { PIF (India) } \\
\text { IBI (India) }\end{array}$ & $\begin{array}{l}=6.630 \\
=1.940 \\
=4.260\end{array}$ \\
\hline
\end{tabular}

При анализе частот показателя D (депрессия) шкалы HADS выявлено, что для женщин с циррозом печени класса C по ChildPugh и у мужчин с циррозом печени класса В по Chilg-Pugh типичным признаком является субклинически выраженная депрессия $(\mathrm{p}<0,05)$, для пациенток с алкогольным стеатогепатитом клинически выраженная депрессия $(\mathrm{p}<0,05)$. Для женщин с циррозом печени класса А по ChildPugh c равной вероятностью можно встретить состояния клинически выраженной депрессии и её отсутствие ( $>>0,05)$, с классом В по Child-Pugh - субклинически выраженной депрессии и её отсутствие $(\mathrm{p}>0,05)$. У пациенток с ХВГ с равной вероятностью можно встретить субклинически и клинически выраженную депрессию ( $>0,05)$, с неалкогольным стеатогепатитом - все три состояния $(\mathrm{p}>0,05)$. Показатели уровня депрессии у женщин представлены на рисунке 3, у мужчин - на рисунке 4 (степень выраженности депрессии указана в условных единицах).

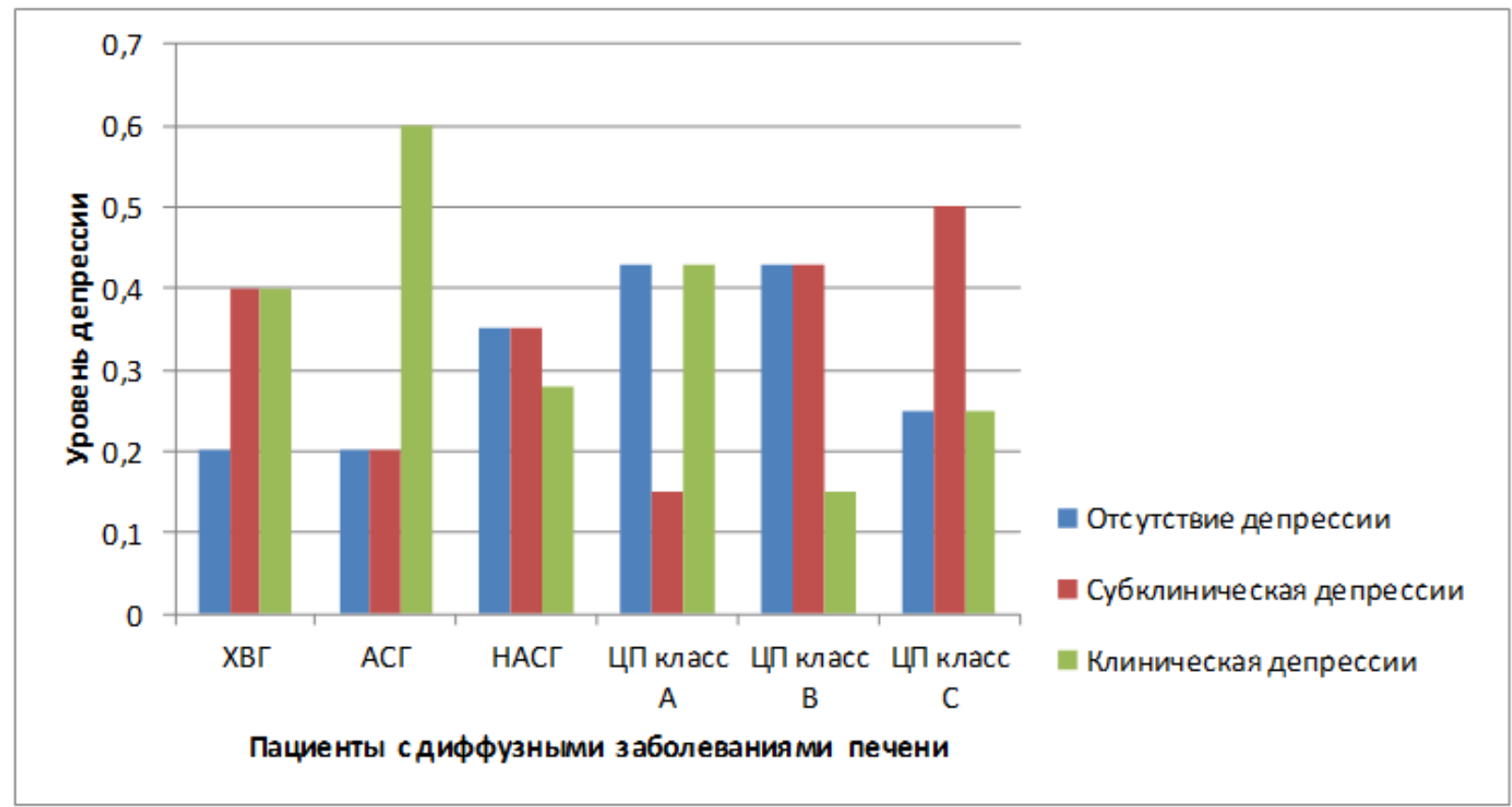

Рисунок 3 - Показатели депрессии у женщин.

Отсутствие депрессии и различная степень её выраженности с равной вероятностью может встречаться у мужчин с ЦП класса A по ChildPugh, стеатогепатитом алкогольной и неалкогольной этиологии и ХВГ (p>0,05). У пациентов мужского пола с ЦП класса С по Child-Pugh можно встретить субклинически выраженную депрессию и её отсутствие $(\mathrm{p}>0,05)$. 


\begin{tabular}{l|lrl|l|ll} 
& ISRA (India) & $=\mathbf{1 . 3 4 4}$ & SIS (USA) & $=\mathbf{0 . 9 1 2}$ & ICV (Poland) & $=\mathbf{6 . 6 3 0}$ \\
Impact Factor: & ISI (Dubai, UAE) $=\mathbf{0 . 8 2 9}$ & PUHL (Russia) $=\mathbf{0 . 2 3 4}$ & PIF (India) & $=\mathbf{1 . 9 4 0}$ \\
& GIF (Australia) & $\mathbf{0 . 5 6 4}$ & ESJI (KZ) & $=\mathbf{1 . 0 4 2}$ & IBI (India) & $=\mathbf{4 . 2 6 0}$ \\
& JIF & $\mathbf{1 . 5 0 0}$ & SJIF (Morocco) & $=\mathbf{2 . 0 3 1}$ & & \\
\hline
\end{tabular}

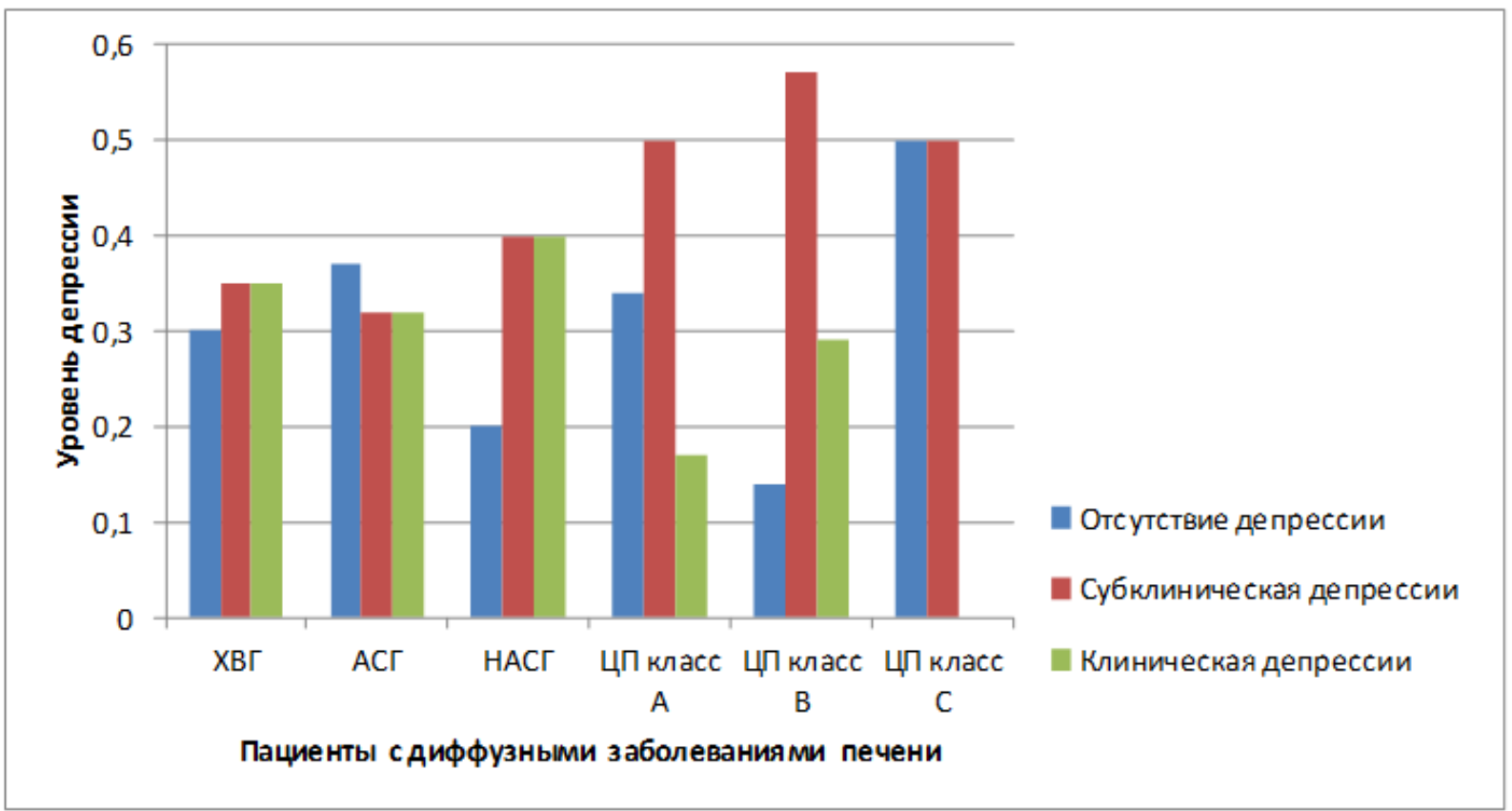

Рисунок 4 - Показатели депрессии у мужчин.

Выводы: Таким образом, согласно результатам опросника HADS, отсутствие тревоги является типичным признаком для пациентов мужского пола с неалкогольным стеатогепатитом. Субклинически выраженная тревога чаще встречается у женщин с алкогольным стеатогепатитом, циррозом печени класса А и класса В по Child-Pugh, а так же у мужчин с алкогольным стеатогепатитом и циррозом печени класса А по Child-Pugh.
Клинически выраженная тревога типична для пациенток с хроническим вирусным гепатитом. Субклиническая депрессия является типичным признаком для женщин, страдающих циррозом печени класса C по Child-Pugh и для пациентов мужского пола с циррозом печени класса В по Child-Pugh. Клиническая депрессия характерна для пациенток с алкогольным стеатогепатитом.

\section{References:}

1. Blum F, Leyzerson A, Khofstedter L (1988) Mozg, razum i povedenie: per. s angl. - M.: Mir, 1988. - 248 p.

2. German E, Prusinski A (1969) Nevrologicheskie sindromy v klinike vnutrennikh bolezney / - Varshava : Polskoe gosudarstvennoe meditsinskoe izd-vo, 1969. — $218 \mathrm{p}$.

3. Organov RG, et al. (2004) Depressii i rasstroystva depressivnogo spektra V obshchemeditsinskoy praktike. Rezultaty programmy KOMPAS // Kardiologiya. 2004. - №1. - pp. 48-49.

4. Nikulina MA (2010) Sovershenstvovanie metodov diagnostiki i lecheniya zabolevaniy gepatobiliarnoy sistemy $\mathrm{s}$ uchetom psikhosomaticheskogo statusa patsientov: avtoref. dis. ... d-ra med. nauk. - Barnaul, 2010. $-45 \mathrm{p}$.

5. Smulevich AB (2001) Depressii v obshchey meditsine - M.: Izd-vo MIA, 2001. - pp. 1624.

6. Smulevich AB (2003) Depressii pri somaticheskikh i psikhicheskikh zabolevaniyakh - M.: Izd-vo MIA, 2003. pp. 155-157.

7. Alekseeva AS (2009) Kliniko-morfologicheskie proyavleniya khronicheskikh gepatitov i tsirrozov pecheni razlichnoy etiologii vo vzaimosvyazi s somatopsikhicheskimi narusheniyami i kachestvom zhizni patsientov: avtoref. dis. ... d-ra med. nauk: - Tomsk, 2009. $-42 \mathrm{p}$. 


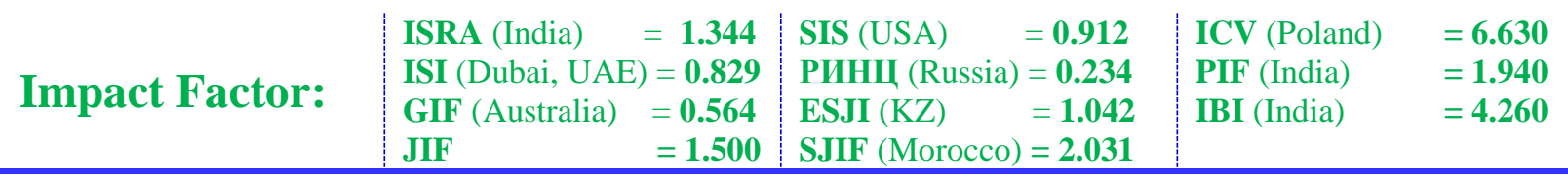

8. Babkin DI (2001) Psikhicheskie narusheniya u bolnykh khronicheskimi diffuznymi zabolevaniyami pecheni: - M., 2001. - 165 p.

9. Yerokhina LG, Gubskiy LV (1990) Nervnopsikhicheskie rasstroystva pri portalnoy gipertenzii // Aktualnye problemy somatopsikhiatrii i psikhosomatiki. - M., 1990 — pp 81-83.

10. Karpinchik NF (1989) Medikopsikhologicheskoe issledovanie bolnykh $\mathrm{s}$ khronicheskimi gepatitami i tsirrozami pecheni: avtoref. dis... kand. med. nauk : — L., 1989. $20 \mathrm{p}$.

11. Alekseeva AS, et al. (2006) Psikhosomaticheskie rasstroystva u bolnykh s khronicheskimi zabolevaniyami pecheni // Sibirskiy vestnik gastroenterologii i gepatologii. - 2006. — № 20. - pp. 134-137.

12. Revenyuk LI, Zavilyanskaya YN (1974) Psikhoterapiya v kompleksnom lechenii zabolevaniy pecheni i zhelchevyvodyashchikh putey // Voprosy meditsinskoy deontologii i psikhoterapii. - Tambov, 1974. - pp. 266269.

13. (2009) Somatonevrologiya: rukovodstvo dlya vrachey / pod red. A. A. Skoromtsa. - SPb. : SpetsLit, 2009. - 655 p.

14. Khomutov AY, Kulba SN (2008) Anatomiya tsentralnoy nervnoy sistemy: uchebnoe posobie — Izd. 4-e. — Rostov n/D : Feniks, 2008. $315 \mathrm{p}$.

15. Aleksandrovskiy YB (1993) Pogranichnye psikhicheskie rasstroystva - M.: Meditsina, 1993. - $400 \mathrm{p}$.

16. Moris V, Ropper AK (2006) Rukovodstvo po nevrologii po Adamsu i Viktoru. (per. s angl.) - 7-e izd. - M. : OOO «Meditsinskoe informatsionnoe agenstvo», 2006. - pp. 466467.

17. (2012) Nevrologiya. Natsionalnoe rukovodstvo. / Pod red. Ye.Guseva - M. : GEOTAR-Media, 2012. - 1080 p.

18. (1998) Psikhiatriya.: / Pod red. Ye.Sheyder. (per. s angl.) - M. : Praktika, 1998. - 485 p. 